\title{
Autoimmune encephalopathy with parasomnia and obstructive sleep apnea
}

INSERM

\section{Source}

INSERM. (1999). Orphanet: an online rare disease and orphan drug data base.

Autoimmune encephalopathy with parasomnia and obstructive sleep apnea.

ORPHA:420789

Autoimmune encephalopathy with parasomnia and obstructive sleep apnea is a rare neurologic disorder characterized by a unique non-REM and REM parasomnia with sleep breathing dysfunction, gait instability and repetitive episodes of respiratory insufficiency, as well as autoantibodies against IgLON5. Patients may present stridor, chorea, limb ataxia, abnormal ocular movements, and bulbar symptoms (i.e. dysphagia, dysarthria, episodic central hypoventilation) with normal brain MRI. Excessive day sleepiness and cognitive deterioration have also been reported. 